\title{
Comparison of laparoscopic and abdominal methods of hysterectomy from patient's perspective
}

\author{
Hasan Terzi ${ }^{1}$, Pinar Solmaz Hasdemir ${ }^{2 *}$, Sertan Aksu ${ }^{1}$, Ahmet Kale ${ }^{1}$, Semra Oruc Koltan ${ }^{2}$
}

\begin{abstract}
Objective: The aim of this study was to compare the postoperative pain and satisfaction of patients who underwent total laparoscopic or abdominal hysterectomy for benign gynecologic conditions.

Materials and Methods: This study was a prospective, randomized trial. A visual analogue scale and patient satisfaction score scale were used to evaluate the patients' postoperative satisfaction rates. Seventy-one patients who underwent total laparoscopic hysterectomy were compared with 68 patients who underwent total abdominal hysterectomy for benign gynecologic indications.

Results: The groups were similar with respect to age, race, gravidity and parity status, and uterine weight. Hospital stay; need for analgesic use; visual analogue scale pain scores at 12, 24, and 36 hours; patient satisfaction scores at 24 and 48 hours and one week; and blood loss were statistically lower in the laparoscopic hysterectomy group than in the abdominal hysterectomy group $(\mathrm{p}<0.001)$.

Conclusion: Laparoscopic hysterectomy was superior to abdominal hysterectomy in terms of short-term followup, postoperative pain, and satisfaction with the operation scar.
\end{abstract}

Key words: Abdominal Hysterectomy, Laparoscopic hysterectomy, Pain, Satisfaction, Visual Analog Scala

\section{Introduction}

More than $70 \%$ of hysterectomies are performed for benign surgical indications, including fibroids (33\%), uterine prolapse $(28 \%)$, menorrhagia $(21 \%)$, and pelvic pain (3\%) (1). The first total laparoscopic hysterectomy was reported in 1989; this procedure has been associated with shorter hospital stay, faster recovery, and fewer postoperative infections compared with abdominal hysterectomy (2). Advanced laparoscopic procedures are increasingly being utilized in gynecologic surgery (3); however, the abdominal hysterectomy technique is still performed in over $80 \%$ of operations (4).

The visual analogue scale (VAS) is a psychometric response scale that can be used in questionnaires. It is a measurement instrument for subjective characteristics or attitudes that cannot be measured directly. When responding to a VAS item, respondents specify their level of agreement with a statement by indicating a position along a continuous line between two end-points. This continuous (or analogue) aspect of the scale differentiates it from discrete scales. There is evidence showing that visual analogue scales have metric characteristics that are superior to those of discrete scales; thus, a wider range of statistical methods can be applied to the measurements (5). The patient satisfaction score (PSS) is a similar scoring system that calculates the satisfaction of the patient in a similar manner as VAS.

The aim of this study was to compare the short-term results of the laparoscopic and abdominal hysterectomy techniques and to compare the satisfaction rates of the patients with the operation scar.

\section{Materials and Methods}

This study was designed as a prospective, randomized trial. Seventy-one patients who underwent total laparoscopic hysterectomy were compared with 68 patients who underwent total abdominal hysterectomy for benign gynecologic indications. The indications for operation were fibroid, abnormal uterine bleeding, endometrial hyperplasia, and cervical intraepithelial neoplasia. Age, weight, and height of the patients were recorded just prior to going into the operating room. A metric body mass index (BMI) calculator was used to calculate the BMIs of the patients. Postoperative pain was measured on a VAS at 12, 24, and 36 hours postoperatively. The patients were asked to rate their pain on a scale of $1-10(0=$ no pain; $2=$ mild; $5=$ moderate; $7=$ severe; $10=$ excruciating). The patients were also evaluated for their satisfaction with 
the abdominal operation scar on a PSS scale at postoperative 24 and 48 hours and one week. The patients were asked to rate their satisfaction with the operation scar on a scale of 1-10 (1=minimum and $10=$ maximum). All of the patients in the trial were evaluated by the same nursing staff.

Approval was obtained from an independent ethics committee, and the patients provided formal, informed consent prior to their participation in the clinical study.

Statistical analyses were carried out using PASW software, version 15.0 for Windows (SPSS, Chicago, IL). Student's t-test was used for statistical comparisons.

\section{Results}

The groups were similar in terms of age, race, gravidity and parity status, and uterine weight. The mean BMI value was statistically lower in the laparoscopic group than in the open abdominal group $(27.65 \pm 4.07$ and $29.53 \pm 3.90$, respectively; $p=0.006)$. There was no need to convert from laparoscopic to open abdominal surgery in any of the cases.

Hospital stay; need for analgesic use; VAS pain scores at 12, 24, and 36 hours; patient satisfaction scores at 24 and 48 hours and one week; and blood loss were statistically lower in the laparoscopic hysterectomy group than in the abdominal hysterectomy group $(p<0.001)$. Although not statistically significant, the operation time was shorter in the laparoscopy group (Table 1). The indications for the operation are shown in Table 2.

Six $(8.5 \%)$ patients in the laparoscopic hysterectomy group and $45(66.2 \%)$ patients in the abdominal hysterectomy group had a history of abdominal operation $(p<0.001)$. No serious perioperative complications were observed in either group. Only one uncomplicated wound infection occurred, in the abdominal hysterectomy group.

\section{Discussion}

Because hysterectomy is a frequent surgical procedure in gynecology, gynaecologists continuously research improved alternative techniques, and advanced laparoscopic techniques have been increasingly used in gynaecologic surgery over the past 20 years.

Previous studies have shown that laparoscopic hysterectomy is a comparable method to abdominal hysterectomy and results in less blood loss, shorter hospital stay, fewer wound infections, less pain, quicker recovery, and better short-term quality of life results. In those studies, mean operation time was longer in the laparoscopy groups $(6,7,8)$. Another study that compared laparoscopic and abdominal hysterectomies found that the operation time was significantly longer in the laparoscopy group, estimated perioperative bleeding was greater in the abdominal hysterectomy group, and there was no difference in length of postoperative hospital stay between the two groups (9). Our study found that both operation time and hospital stay were shorter in the laparoscopic hysterectomy group. This difference might be due to the surgeons' experience with laparoscopic procedures in our study center. Although no major perioperative complications were observed in our study population, lower complication rates have been reported with laparoscopic procedures in the literature $(10,11)$.

There are two novel reports comparing laparoscopic hysterectomy with mini laparotomic abdominal hysterectomy. In a retrospective analysis, Kumar et al. found that mini laparotomy had a shorter intraoperative time and less blood loss, but a higher rate of major wound complications (12). Sirisabya et al. found similar postoperative pain and patient satisfaction results in the two groups, but a much higher postoperative complication rate in the laparoscopy group (13). These reports are not consistent with our findings. Although the differences might be related to the experience of the surgeons and the center or to the mini laparotomic incision in the abdominal approach, further studies are needed.

A study comparing laparoscopic and abdominal hysterectomies in terms of quality of life in a small study group found a significant treatment effect favoring laparoscopic hysterectomy in the RAND-36 scale for vitality (14).

Postoperative pain and the appearance of the operation scar are two valuable parameters for hysterectomy patients. In our study, postoperative pain and need for analgesic use were lower in the laparoscopy group, which is similar to results found in the literature. We also asked the patients what they thought of their operation scar, and the satisfaction rate was significantly higher in the laparoscopic group. We believe this is an important parameter when choosing the operative technique.

\section{Conclusion}

In conclusion, total laparoscopic hysterectomy is a remarkable alternative operation to abdominal hysterectomy in the management of benign gynecologic conditions when the operation team is experienced with laparoscopic surgery. Minimally invasive techniques could improve patient satisfaction and compliance with the operation.

\section{Acknowledgements}

All authors state that there is no conflict of interest 
Table 1. Group statistics of the laparoscopic and abdominal hysterectomy patients

\begin{tabular}{lccc} 
& $\begin{array}{c}\text { Laparoscopy } \\
\text { group (n=71) }\end{array}$ & $\begin{array}{c}\text { Abdominal Hysterectomy } \\
\text { group (n= 68) }\end{array}$ & p-value \\
\hline Age & $47,7 \pm 5,13$ & $47,44 \pm 4,63$ & 0,752 \\
BMI & $27,65 \pm 4,07$ & $29,53 \pm 3,90$ & 0,006 \\
Gravidy & $3,32 \pm 1,39$ & $3,73 \pm 2,39$ & 0,216 \\
Parity & $2,70 \pm 1,17$ & $2,78 \pm 1,19$ & 0,709 \\
Uterine weight (g) & $387,60 \pm 113,64$ & $385,51 \pm 102,0$ & 0,909 \\
Preop. Hb level & $10,99 \pm 0,90$ & $11,17 \pm 0,87$ & 0,252 \\
Postop. Hb level & $10,14 \pm 0,91$ & $10,08 \pm 0,74$ & 0,666 \\
Hospital stay & $3,21 \pm 0,61$ & $4,29 \pm 0,95$ & $\mathbf{0 , 0 0 0}$ \\
Analgesic need & $2,66 \pm 0,97$ & $6,60 \pm 0,59$ & $\mathbf{0 , 0 0 0}$ \\
VAS 12 hours & $5,12 \pm 1,51$ & $7,07 \pm 1,15$ & $\mathbf{0 , 0 0 0}$ \\
VAS 24 hours & $3,98 \pm 1,11$ & $5,57 \pm 1,02$ & $\mathbf{0 , 0 0 0}$ \\
VAS 36 hours & $3,28 \pm 0,99$ & $4,14 \pm 1,02$ & $\mathbf{0 , 0 0 0}$ \\
PSS 24 hours & $8,43 \pm 7,77$ & $5,75 \pm 1,02$ & $\mathbf{0 , 0 0 0}$ \\
PSS 48 hours & $9,07 \pm 0,54$ & $6,55 \pm 1,09$ & $\mathbf{0 , 0 0 0}$ \\
PSS 1 week & $9,49 \pm 0,53$ & $7,05 \pm 1,23$ & $\mathbf{0 , 0 0 0}$ \\
Blood loss (ml) & $118,45 \pm 79,16$ & $212,64 \pm 162,87$ & $\mathbf{0 , 0 0 0}$ \\
Operation time (min) & $86,12 \pm 18,97$ & $93,19 \pm 22,15$ & 0,062 \\
\hline
\end{tabular}

BMI: Body-mass index, $\mathrm{p}<0,005$, VAS: Visual Analoque Scala, PSS: Patient Satisfaction Score

Table 2. Distribution of the operation indications of the patients

\begin{tabular}{lccc}
\hline \multicolumn{3}{c}{ Group } \\
\hline Diagnosis & $\begin{array}{c}\text { Laparoscopy } \\
(\mathbf{n}=\mathbf{7 1})\end{array}$ & $\begin{array}{c}\text { Abdominal } \\
(\mathbf{n}=\mathbf{6 8})\end{array}$ & $\begin{array}{c}\text { Total } \\
(\mathbf{n}=\mathbf{1 3 9})\end{array}$ \\
\hline Myoma & $31(\% 43,7)$ & $31(\% 45,6)$ & $62(\% 44,6)$ \\
Abnormal uterine bleeding (AUB) & $14(\% 19,7)$ & $14(\% 20,6)$ & $28(\% 20,1)$ \\
Myoma+ AUB & $18(\% 25,4)$ & $18(\% 26,5)$ & $36(\% 25,9)$ \\
Endometrial hyperplasia & $7(\% 9,8)$ & $5(\% 7,4)$ & $12(\% 8,6)$ \\
Cervical Intraepithelial Neoplasia & $1(\% 1,14)$ & $0(\% 0)$ & $1(\% 0,7)$ \\
\hline
\end{tabular}

\section{References}

1- Brummer THI, Jalkanen J, Fraser J. National Prospective 1-Year Survey of 5279 Hysterectomies. Hum Reprod. 2009;10:2515-2522

2- Reich H, De Caprio J, Mc Glynn F. Laparoscopic Hysterectomy. J Gynecol Surgery. 1989;5:213- 216.

3- Falcone T, Paraiso MF, Mascha E. Prospective randomized clinical trial of laparoscopically assisted vaginal hysterectomy versus total abdominal hysterectomy. Am J Obstet Gynecol. 1999;180(4):955-62

4- Candiani M, Izzo S. Laparoscopic versus vaginal hysterectomy for benign pathology. Curr Opin Obstet Gynecol. 2010;22(4):304-8.

5- Reips and Funke F . "Interval level measurement with visual analogue scales in Internet-based research: VAS Generator." 2008. doi:10.3758/BRM.40.3.699

6- Johnson N, Barlow D, Lethaby A, Tavender E, Curr E, Garry R. Surgical approach to hysterectomy for benign gynaecological disease. Cochrane Database Syst Rev. 2006;19(2):CD003677.

7- Garry R, Fountain J, Brown J, Manca A, Mason S, Sculpher $M$, et al. Evaluate hysterectomy trial: a multicentre randomised trial comparing abdominal, vaginal and laparoscopic methods of hysterectomy. Health Technol Assess. 2004:8(26):1-154

8- Jahan S, Das T.R, Mahmud N, Mondol S.K, Habib S.H, Saha S, et al. A Comparative study among laparoscopically assisted vaginal hysterectomy, vaginal hysterectomy and abdominal hysterectomy: Experience in a tertiary care hospital in Bangladesh. Journal of Obstetrics and Gynaecology. 2011;31(3):254-257.
9- Oscarsson U, Poromaa IS, Nüssler E, Löfgren M. No difference in length of hospital stay between laparoscopic and abdominal supravaginal hysterectomy--a preliminary study. Acta Obstet Gynecol Scand. 2006;85(6):682-7.

10- Sutasanasuang, S. Laparoscopic hysterectomy versus total abdominal hysterectomy: A retrospective comparative study. Journal of the Medical Association of Thailand 2011;94(1):8-16

11- Wiser, A., Holcroft, C.A., Tulandi, T., Abenhaim, H.A. Abdominal versus laparoscopic hysterectomies for benign diseases: Evaluation of morbidity and mortality among 465,798 cases. Gynecological Surgery 2013;10(2):117122

12- Kumar A, Pearl M. Mini-laparotomy versus laparoscopy for gynecologic conditions. J Minim Invasive Gynecol. 2014;21(1):109-14

13- Sirisabya N, Manchana T. Mini-laparotomy vs laparoscopic hysterectomy for benign gynaecological diseases. J Obstet Gynaecol. 2014;34(1):65-9.

14- Kluivers KB, Hendriks JC, Mol BW, Bongers MY, Bremer $\mathrm{GL}$, de Vet HC, et al. Quality of life and surgical outcome after total laparoscopic hysterectomy versus total abdominal hysterectomy for benign disease: a randomized, controlled trial. J Minim Invasive Gynecol. 2007;14(2):145-52.

Copyright (C) 2014 The Author(s); This is an open-access article distributed under the terms of the Creative Commons Attribution License (http://creativecommons.org/licenses/by/4.0), which permits unrestricted use, distribution, and reproduction in any medium, provided the original work is properly cited. 